\title{
Unusual Presentation of metastasis in Spine.
}

\author{
Dr Atul K Patil ${ }^{1}$, Dr Pathak Aditya $C^{2}$, Dr Sanjay Jadhav ${ }^{3}$,Dr Anil Khandekar ${ }^{4}$. \\ ${ }^{1-}$ Associate Professor, Department of Orthopaedics, Terna Medical College and Hospital, Sector - 12, Phase - \\ 2, Nerul West, Navi Mumbai, Mumbai, Maharashtra 400706. \\ 2- Assistant Professor, Department of Orthopaedics, Dr RN Cooper Muncipal General Hospital, Vile parle- \\ west, Mumbai 400056. \\ 3- Professor, Head of Department of Orthopaedics, Terna Medical College and Hospital, Sector - 12, Phase - 2, \\ Nerul West, Navi Mumbai, Mumbai, Maharashtra 400706. \\ 4- Professor, Department of Orthopaedics, Terna Medical College and Hospital, Sector - 12, Phase - 2, Nerul \\ West, Navi Mumbai, Mumbai, Maharashtra 400706.
}

\begin{abstract}
In elderly age group metastasis to spine is a known occurrence and so is prevalence of Koch's spine in developing country like India where tuberculosis is a very common occurrence. Both have similar clinical and radiological occurrence. Hence it is must to have tissue diagnosis before executing the treatment. Here we present a very unusual presentation of adenocarcinoma of lungs with metastasis to spine and having striking clinical and radiological resemblance to tuberculosis
\end{abstract}

Keywords: Adenocarcinoma, Tuberculosis, Spine, Metastasis.

\section{Introduction}

The association of TB with carcinoma was initially described about 200 years ago by Bayle who considered 'cavitation cancereuse' as one of the various types of TB. ${ }^{1}$ Tuberculosis infection is seen with increasing frequency both in developed as well as undeveloped countries. Although this infection is usually confined to the respiratory system, spinal involvement is not uncommon and occurs in approximately $1 / 5 \%$ of all infected cases. ${ }^{2}$ About $10 \%$ of all cancer patients develop metastases to the spine. Among immunocompetent adult patients with cancer, $60 \%$ of these spinal metastases are either from the breast, lung, or prostate. ${ }^{3}$ In this case report we present a unusual presentation of adenocarcinoma metastasis to posterior spinous process of dorsal vertebrae with abscess evident on surgery mimicking tuberculosis.

\section{Case Report-}

A 70 year old male patient presented in the outpatient department with severe backache and subacute onset weakness in both lower limbs. Patient was apparently alright 1 months back after which he started having cough, haemoptysis(2-3 episodes), loss of weight and loss of appetite. After symptomatic treatment patient cough decreased but was persistent and haemoptysis settled. Patient also started experiencing back pain which was continuous, decreased partially with medication and increased in evening hours. Patient neglected the back pain. 2 weeks after back pain patient developed clumsiness of gait and later inability to walk and then patient was bedridden since last 1 week with Grade 3 power in both lower limbs. Patient had a positive history of Kochs contact and had no other significant past medical or surgical illness. Hence patient was evaluated for Tuberculosis. Sputum AFB came positive along with significant "Montoux test". Chest roentogram shows prominent lung markings with opacity in both apical lobes and prominent Hilar markings. There was no Lymphadenopathy. Rest systemic examination was normal and patient had no significant personal history. Patient had no bowel bladder sensations. On spine examination, patient had spasticity with exaggerated reflexes, extensor plantars and paraparesis with sensations below D7 decreased bilaterally by 50 percent and absent superficial reflexes. There was tenderness on spine examination at D5- D8 level. Magnetic resonance scan of spine was done. It showed focal lesion in posterior column of D5 vertebrae with destruction of posterior elements with granulation tissue in spinal cord compressing it most likely to be secondary to Tuberculosis. Patient was operated from posterior transpedicular approach. Posterior diseased elements of vertebrae were debrided, and abscess around the spinal cord was removed thus decompressing the cord decompressed. Surgery was uneventful and AKT Category 1 was started. . Collected granulation Tissue and sample was sent for BACTEC, culture and Histopathology. To our dismay all reports came negative for Tuberculosis and unfortunately histopathology report concluded poorly differentiated Adenosquamous carcinoma most likely metastatic. On further workup and reports it was then found that patient had adenoaquamous carcinoma of lungs and treatment was given accordingly.

Patient is now at 6 months follow up with bladder and bowel sensations recovered and grade 4 power bilaterally in lower limb. 


\section{Discussion-}

Chronic degenerative diseases and cancer are postulated as major causes of morbidity and mortality in geriatric age group. Up to $40 \%$ of cancer patients will develop skeletal metastases; the spine, due to its size, contiguity and rich vascularization, is the primary affected bone site .Among patients who develop spinal metastases, only 5\%-10\% will develop epidural spinal cord compression, and $10 \%$ of those patients will be symptomatic. Incidence of bony metastases increases with prolonged patient survival, and these metastases are derived from primary tumors originating from the kidney, breast, prostate, lungs and other organs. As in our case we had metastasis from lungs but as patient also had Tuberculosis of lungs we had suspected it to have Kochs and its appearance in MRI was also suggestive of Kochs. We have not come across any case with such strange co-existence and resemblance., ${ }^{4,5,6,7}$

Vertebral involvement quantification, vertebral canal invasion, neurological status, general health status and the malignancy prognosis, determined by primary tumor histology, are paramount factors to consider for surgical planning and establishing therapeutic targets. In the spine, the vertebral body is the most common site of metastasis but according to plain radiographs, changes in pedicle is observed at the earliest time, and metastasis development in the posterior element claims about $15 \%$ of the total spinal metastasis. Due to its avascular nature, the disc is usually spared from tumor involvement: however, the most frequently and severely affected part of the vertebra is the vertebral body (in about $80 \%$ ) followed by the pedicles and the posterior elements. Reports have pointed out that about $70 \%$ of symptomatic lesions were found in the thoracic spine, $20 \%$ in the lumbar spine and $10 \%$ in the cervical spine. ${ }^{8}$ On the other hand in tuberculoses vertebral body involvement is most common and spinous process is rarest. In our case the primary site of involvement was spinous process and posterior elements which is rare. ${ }^{4,5}$

Skeletal metastasis of malignant tumor is known to be affected by three specific factors. The first factor is metastasis pathway, which includes the arterial system, direct invasion, lymphatic's, and venous system. Among these four pathways, metastasis via venous system is the most common route of spinal metastasis. Lung cancer can be metastasized directly via segmental artery of spine while breast cancer or prostate cancer can develop spinal metastasis via Batson's ${ }^{4}$ plexus which was most probably the cause in our case.

.In almost $10 \%$ of cases of newly diagnosed spinal metastases, the patient is not known to have cancer, and spinal cord compression is the initial symptom of the malignancy. ${ }^{9}$ The clinical presentation of metastatic spine disease is predominantly pain, neurological deficit, progressive deformity, and general weakness. Pain is the most common presenting symptom in patients with metastatic spinal tumors. The etiology of pain may be tumor spread, spinal instability, nerve root or dural irritation, or direct cauda equina or spinal cord compression. Pain may also be related to bony destruction or invasion of paraspinous tissues, such as muscles or ligaments ${ }^{5}$ .In our case too main presenting feature was pain but there was no evidence of primary malignancy, on the contrary there was evidence of Kochs in Lungs and hence patient was treated as kochs and even MRI feature supported the diagnosis.

Spinal tuberculosis is initially apparent in the anterior inferior portion of the vertebral body. Later on it spreads into the central part of the body or disk. Paradiscal, anterior, and central lesions are the common types of vertebral involvement. Spine is most common site of extrapulmonary tuberculosis. It shows three distinct involvement patterns - paradiscal, central and anterior in descending order. ${ }^{10}$

Such strange presentation and coexistence of Pulmonary Kochs, Adenocarcinoma lung and its Metastasis to spinous process of dorsal vertebrae with abscess present on histopathology is very rare.

To conclude we believe that Metastatic lesion in spine can mimic Tuberculosis in clinical and radiological presentation and hence biopsy is must. ${ }^{11}$ We also support the notion that TB should be taken into consideration in every day clinical practice and included in the differential diagnosis of apparently malignant lesions, at least in endemic regions and in perplexing case. ${ }^{12} \mathrm{We}$ believe irrespective of the age tissue diagnosis either open or CT guided biopsy must be done and then further treatment planning must be executed.

\section{Figure Legends}

I-Preoperative MRI images both sagittal(A)and axial cuts(B) showing destruction of D-5 and D 6 spinous process with minimal cord compression.

II.(A and B)-Intra-operative image of abscess coming from D5 Spinous process.

III. Pet scan images showing Primary and metastatic tumor.

IV- Histopathology report showing adenocarcinoma of lung. 
None

\section{Acknowledgement}

\section{References}

[1]. Pandey M, Abraham E, Chandramohan K, Rajan B. Tuberculosis and metastatic carcinoma coexistence in axillary lymph node: A case report. World J Surg Oncol2003;1:3

[2]. Kapoor SK, Garg V, Dhaon BK, Jindal M: Tuberculosis of the posterior vertebral elements: a rare cause of compression of the cauda equina. J Bone Joint Surg 87A: 391-394, 2005

[3]. J. F. Yegüez, S. A. Martinez, D. R. Sands, L. R. Sands, and M. D. Hellinger, "Colorectal malignancies in HIV-positive patients," American Surgeon, vol. 69, no. 11, pp. 981-987, 2003.

[4]. Akbar M, Ayache A, Eichler M, Klotz M, Wiedenhöfer B, Lehner B. Management of spinal metastases, strategies and surgical indications. Orthopade. 2012;41(8):632-9.[PubMed]

[5]. Shiue K, Sahgal A, Chow E, et al. Management of metastatic spinal cord compression.Expert Rev Anticancer Ther. 2010;10(5):697-708. [PubMed]

[6]. Paton GR, Frangou E, Fourney DR. Contemporary treatment strategy for spinal metastasis: the "LMNOP" system. Can J Neurol Sci. 2011;38(3):396-403. [PubMed]

[7]. Choi D, Crockard A, Bunger C, et al. Global Spine Tumour Study Group. Review of metastatic spine tumor classification and indications for surgery: the consensus statement of the Global Spine Tumour Study Group. Eur Spine J. 2010;19(2):215-22.

[8]. Ufuk AYDINLI, Cagatay OZTURK, Sami BAYRAM et al.- Evaluation of lung cancer metastases to the spine. Acta Orthop. Belg., 2006, 72, 592-597

[9]. Stark RJ, Henson RA, Evans SJ. Spinal metastases :A retrospective survey from a general hospital. Brain $1982 ; 105: 189-213$.

[10]. Abdolwahab IF, Camins MB, Hermann G, Klein MJ: Vertebral arch or posterior spinal tuberculosis: case report. Skeletal Radiol 26:737-740, 1997

[11]. Zamzuri Z,Adham SY,Shukrimi A, Azril MA, Amran R-Metastatic Adenocarcinoma of the Lung Mimicking Spinal Tuberculosis.Volume 10 Number 2, Dec 2011,Page 41-44

[12]. Shinde SR, Chandawarkar RY, Deshmukh SP. Tuberculosis of the breast masquerading as carcinoma: a study of 100 patients. World J Surg 1995;19:379-81.

FIGURE I- A

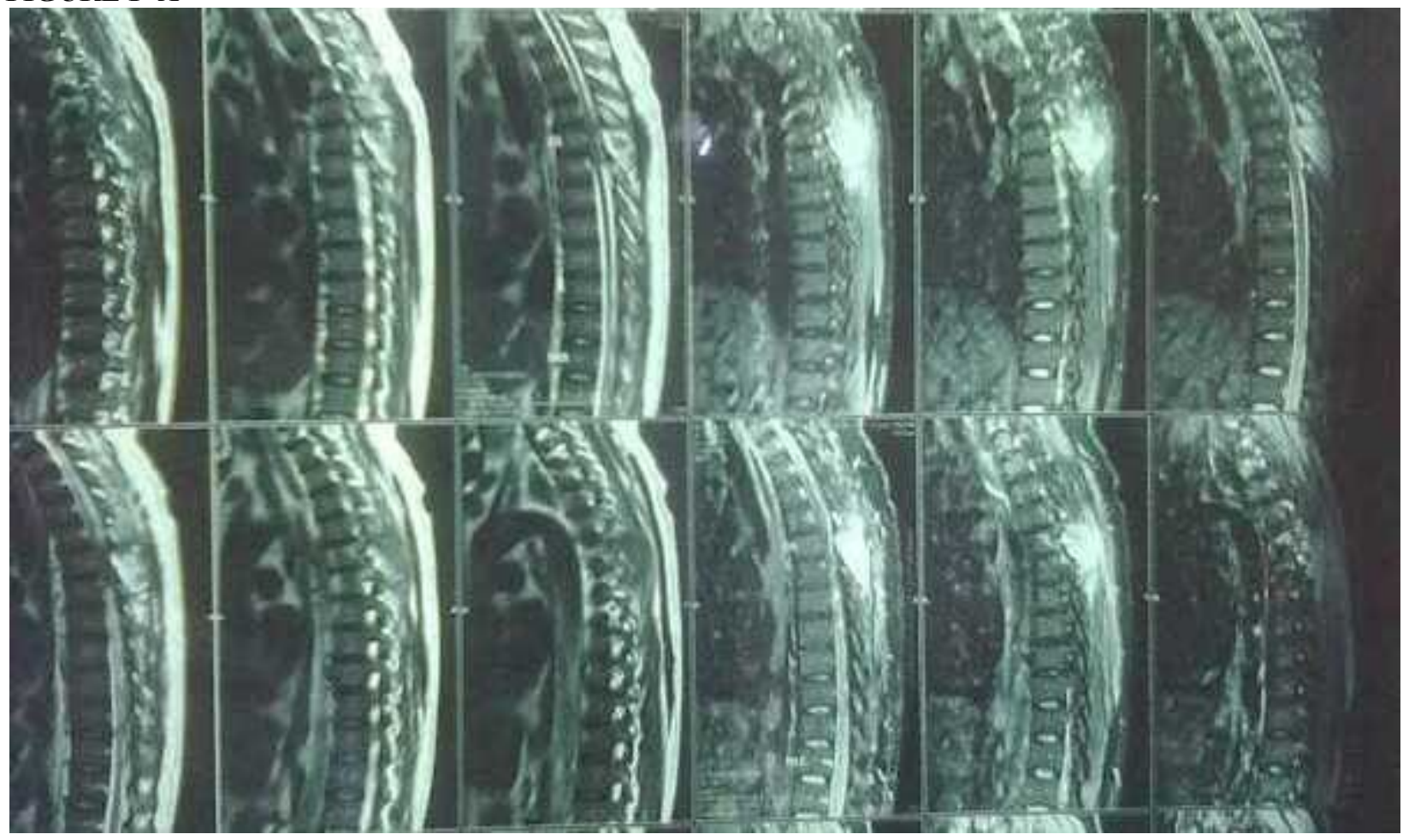




\section{Figure I-B}

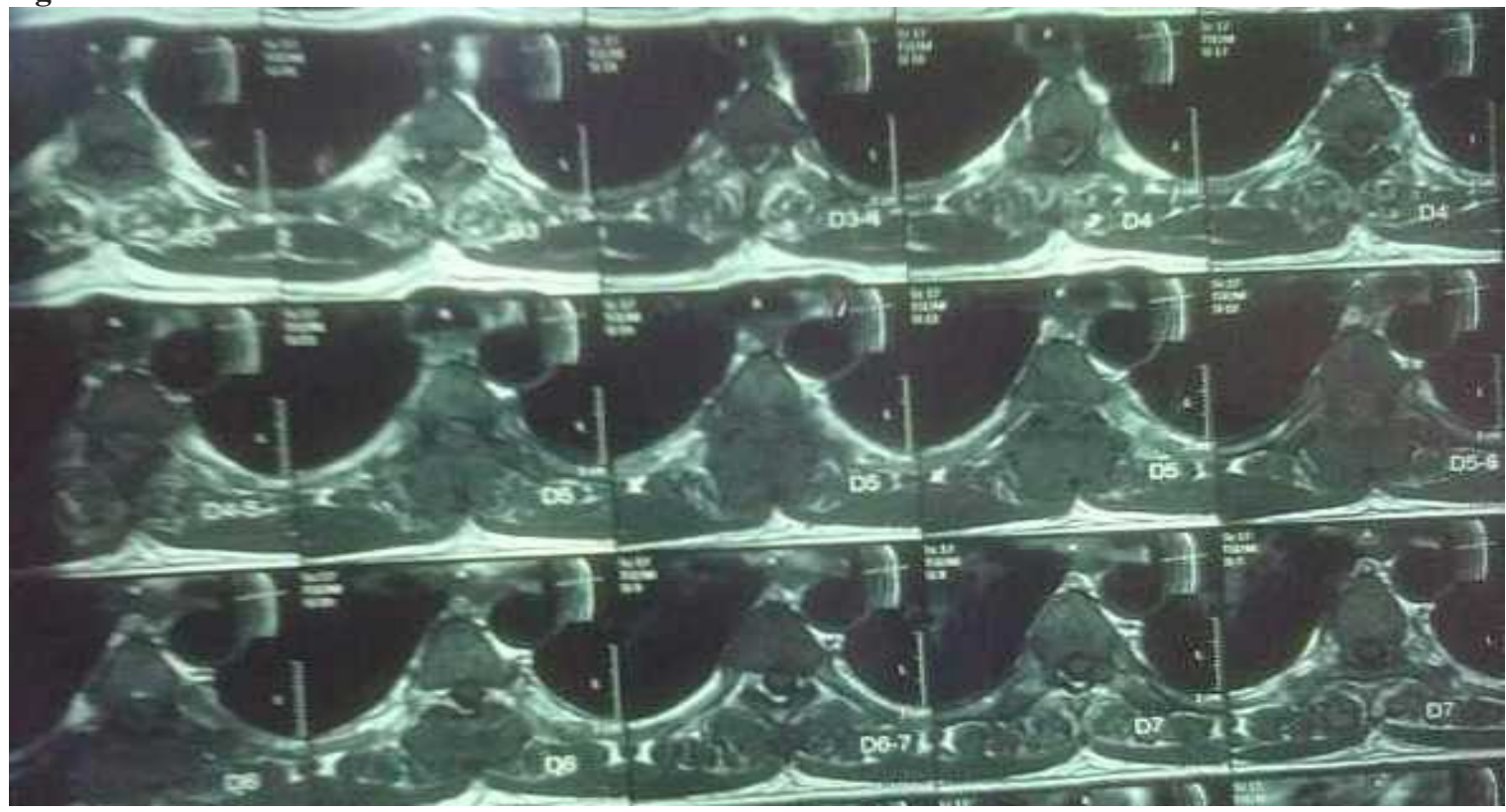

\section{FIGURE II-A}

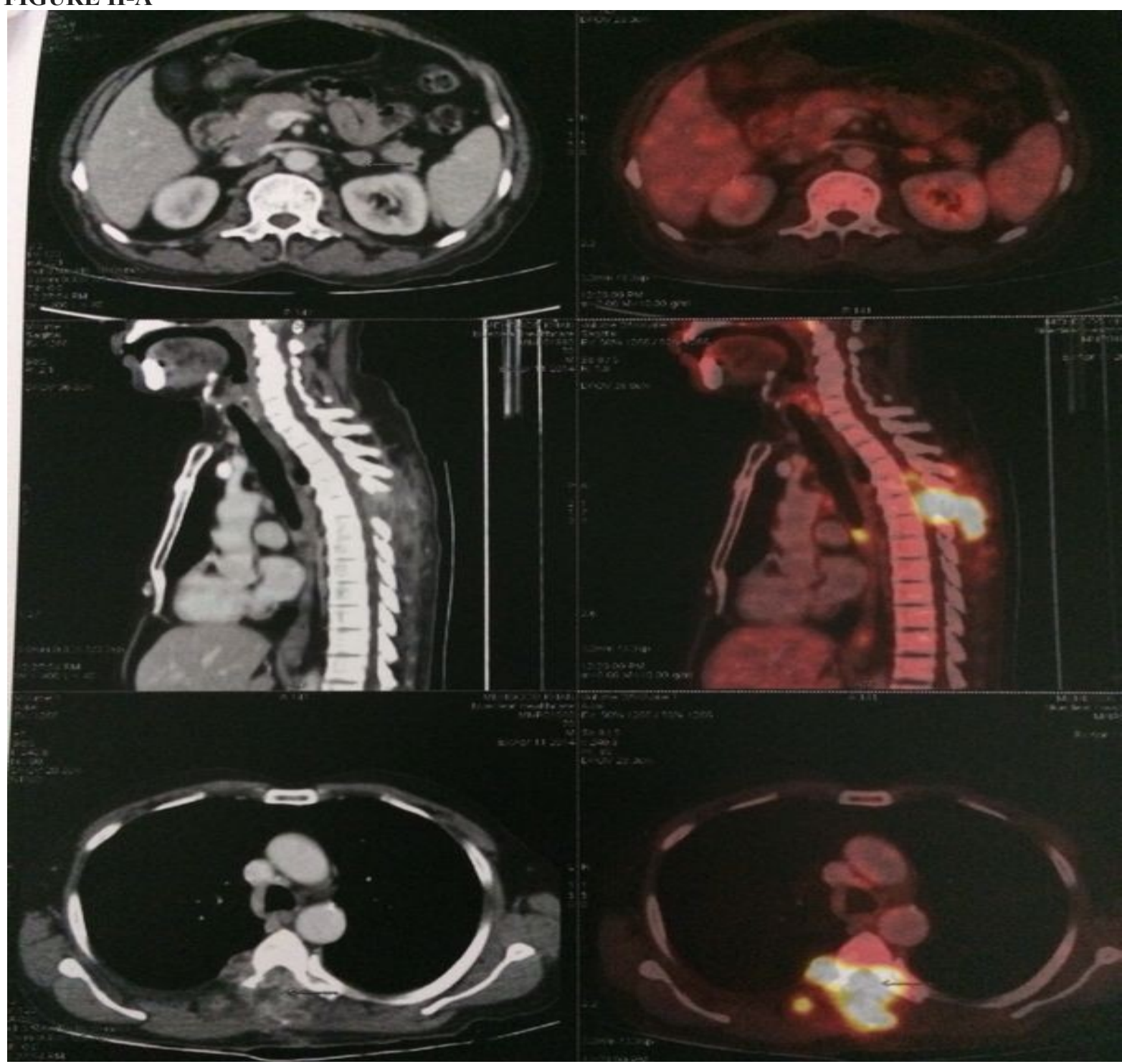




\section{Figure II-B}



Figure III- 


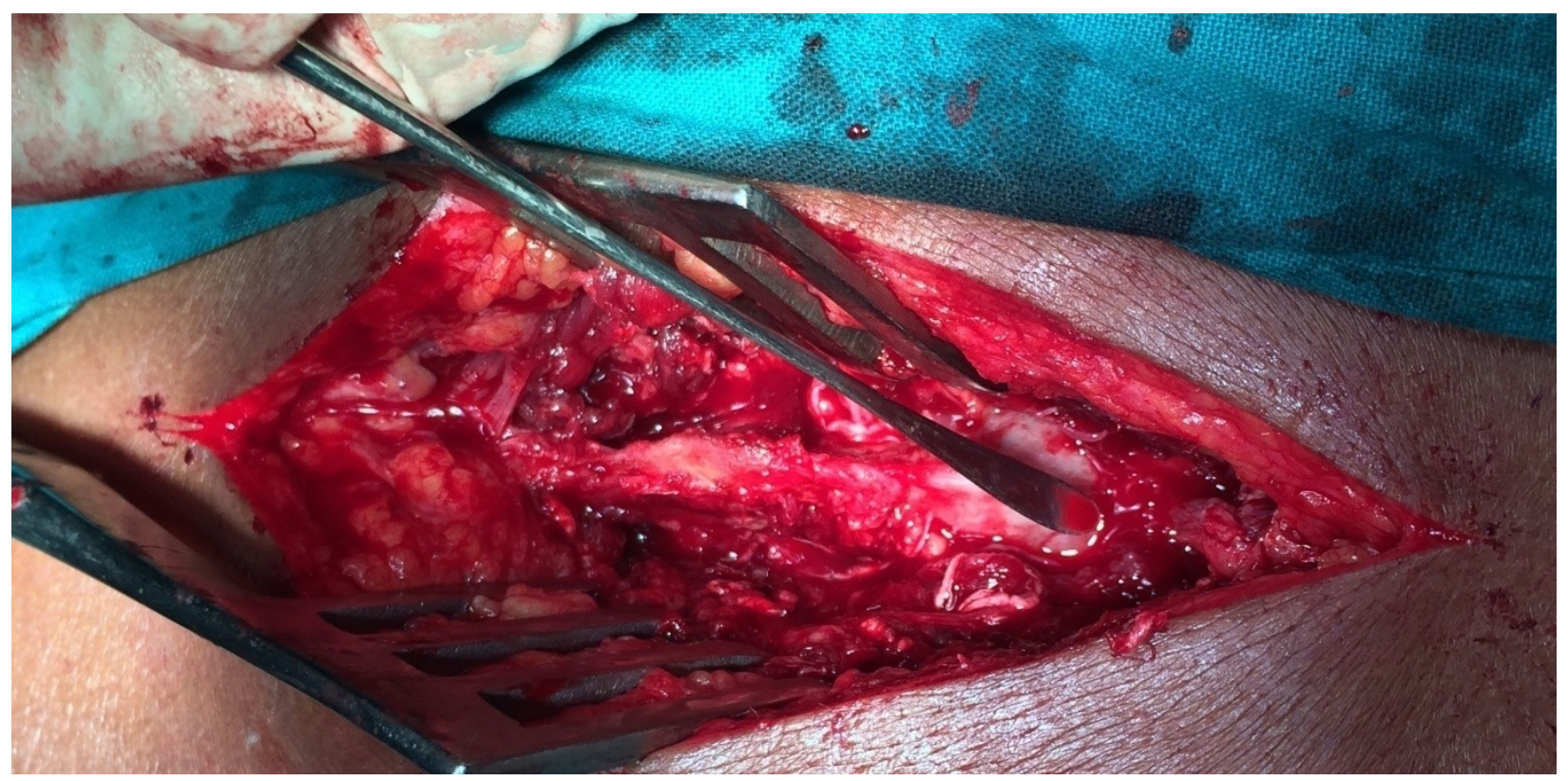

FigureIV-

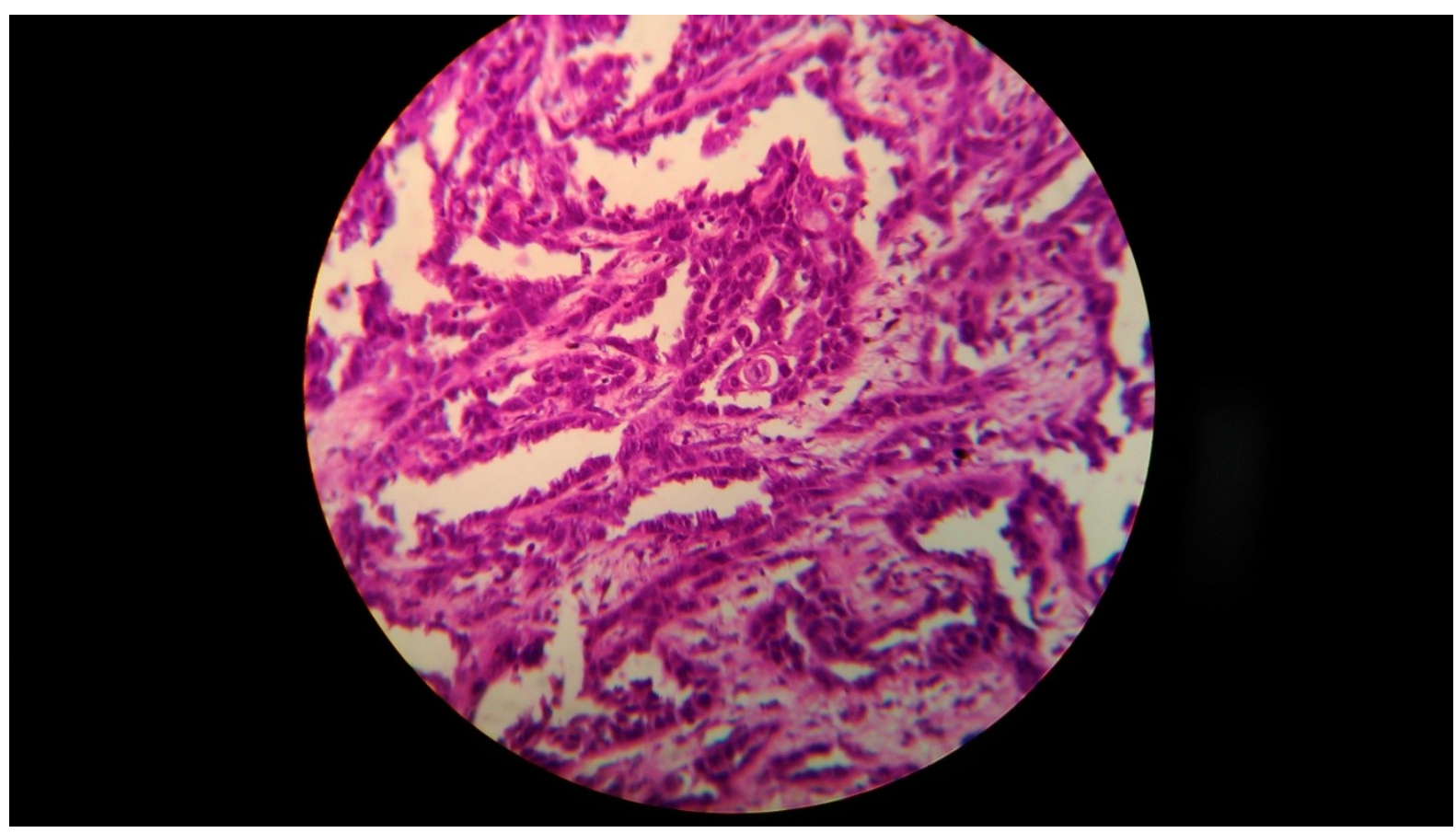

